\title{
Rapid Resolution of Coronary Artery Spasm Complicated by Acute Systolic Heart Failure with Antirejection Therapy in Acute Orthotopic Heart Transplant Rejection
}

Howard Lan*, Liset Stoletniy, Antoine Sakr and Anthony Hilliard

Loma Linda University Medical Center, Loma Linda, CA, USA

\begin{abstract}
We report a case of rapid resolution of Coronary Artery Spasm (CAS) and improvement of systolic heart failure by antirejection therapy with methylprednisolone in the setting of acute Orthotopic Heart Transplant $(\mathrm{OHT})$ rejection. The proposed mechanism of action is reduced microvascular inflammation from steroid therapy resulting in enhanced vasomotor response, resolution of coronary artery spasm, and improved hemodynamic. The case report has treatment implications for patients in acute $\mathrm{OHT}$ rejection with coronary artery spasm. Antirejection therapy with steroid may be helpful in treating life threatening cardiac conditions in patients with acute OHT rejection and coronary artery spasm.
\end{abstract}

Keywords: Antibody mediated rejection; Coronary artery spasm; Orthotopic heart transplant; Systolic heart failure

Abbreviations: AMR: Antibody Mediated Rejection; CAS: Coronary Artery Spasm; HLA: Human Leukocyte Antigen; LAD: Left Anterior Descending; OHT: Orthotopic Heart Transplant; TIMI: Thrombolysis in Myocardial Infarction

\section{Introduction}

Coronary Artery Spasm (CAS) is defined as vasoconstriction of epicardial coronary artery causing vessel narrowing or occlusion [1]. CAS has been described as an early stage of cardiac allograft vasculopathy [2]. CAS involves an imbalance of autonomic nervous system, endothelial dysfunction, and abnormal smooth muscle reactivity [1]. In acute Orthotopic Heart Transplant (OHT) rejection, in addition to attenuated nitric oxide production [3], microvascular inflammation [4] may also be a contributor to CAS. In this case report, we describe the pathophysiology and clinical presentation of a unique case of CAS, in acute OHT rejection, which partially improved with sublingual nitroglycerin but completely resolved after antirejection therapy with steroid within $24 \mathrm{~h}$.

\section{Case Presentation}

29 year-old male with history of dilated cardiomyopathy and OHT two years prior, who complained of dyspnea on exertion, orthopnea, and paroxysmal nocturnal dyspnea for one week. Patient underwent outpatient left and right cardiac catheterization and showed diffuse mid Left Anterior Descending (LAD) narrowing estimated at $75 \%$ throughout (Figure 1) with diagonal involvement, which were not present one year prior. After administration of sublingual nitroglycerin, the stenoses marginally improved (Figure 2) but Thrombolysis in Myocardial Infarction (TIMI) 2 slow flow throughout the coronary bed remained. The Left Ventricular End Diastolic Pressure (LVEDP) was elevated at $26 \mathrm{mmHg}$. Given the patient's history and invasive study findings, he was directly admitted to the cardiac care unit for suspected acute $\mathrm{OHT}$ rejection. While waiting for test results to confirm rejection, patient was started on antirejection immunosuppressive therapy including intravenous methylprednisolone [5-7]. On the following day, patient underwent repeat left cardiac catheterization which showed resolution of the LAD and diagonal stenoses (Figures 3 and 4), normalization of coronary flow (TIMI 3 ), and significantly improved
LVEDP of $14 \mathrm{mmHg}$. Laboratory studies collected on admission revealed the presence of new human leukocyte antigen HLA-DQ, HLA-DQ7, and anti-complement C1q antibodies indicating that the patient had acute antibody mediated rejection (AMR). Echocardiogram showed a reduction in left ventricular ejection fraction from $75 \%$, one month prior, to $45 \%$ with elevated filling pressures. Individualized treatment plan for AMR was escalated to include plasmapheresis $[5,8]$, intravenous immunoglobulin $[5,8]$, and antithymocyte globulin $[9,10]$ infusion. Patient's clinical status improved with OHT antirejection therapy and was discharged home on hospital day ten.

\section{Discussion}

Coronary Artery Spasm (CAS) in OHT has been reported as early as 1981 [11], Buda induced CAS in an OHT patient with intravenous ergonovine maleate. Subsequent reports demonstrated the resolution of CAS in OHT patients by intracoronary nitroglycerin $[12,13]$ and sublingual nitroglycerin [14]. However, we present the first case of CAS in the setting of acute OHT rejection with complete resolution of CAS after initiation of antirejection therapy with methylprednisolone. Secondly, the physiologic response to antirejection therapy was immediate, within $24 \mathrm{~h}$, as evidenced by resolution of CAS and normalization of LVEDP.

The pathophysiology of CAS is complex and involves an imbalance of autonomic nervous system, endothelial dysfunction, and abnormal smooth muscle reactivity [1]. However, patients with OHT are denervated suggesting that autonomic nervous system and cardiac innervation are not essential mechanisms in all CAS [11-14]. At the

*Corresponding author: Howard Lan, Loma Linda University Medical Center, 11234 Anderson Street, Suite 2426, Loma Linda, CA 92354, USA, Tel: +1-909-558-7674; Fax: +1-909-651-5938; E-mail: hwlan@llu.edu

Received November 16, 2016; Accepted November 28, 2016; Published December 05, 2016

Citation: Lan H, Stoletniy L, Sakr A, Hilliard A (2016) Rapid Resolution of Coronary Artery Spasm Complicated by Acute Systolic Heart Failure with Antirejection Therapy in Acute Orthotopic Heart Transplant Rejection. Cardiovasc Pharm Open Access 5: 201. doi: 10.4172/2329-6607.1000201

Copyright: (c) 2016 Lan H, et al. This is an open-access article distributed under the terms of the Creative Commons Attribution License, which permits unrestricted use, distribution, and reproduction in any medium, provided the original author and source are credited. 
Citation: Lan H, Stoletniy L, Sakr A, Hilliard A (2016) Rapid Resolution of Coronary Artery Spasm Complicated by Acute Systolic Heart Failure with Antirejection Therapy in Acute Orthotopic Heart Transplant Rejection. Cardiovasc Pharm Open Access 5: 201. doi: 10.4172/23296607.1000201

Page 2 of 3

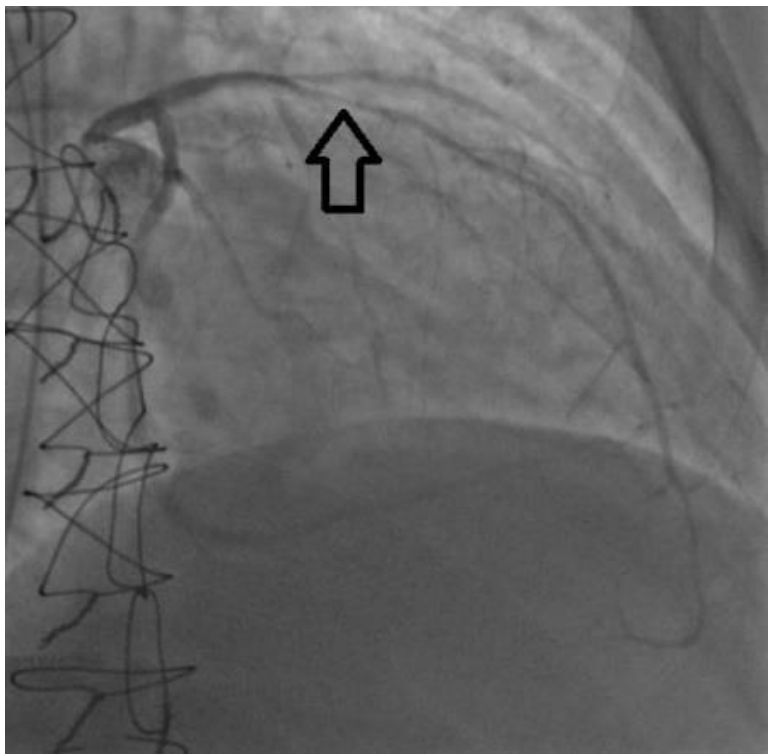

Figure 1: Demonstration of coronary artery spasm in acute orthotopic heart transplant rejection with diffuse mid left anterior descending narrowing with diagonal involvement. Arrow shows the most significant lesion with $75 \%$ stenosis at the mid left anterior descending artery.

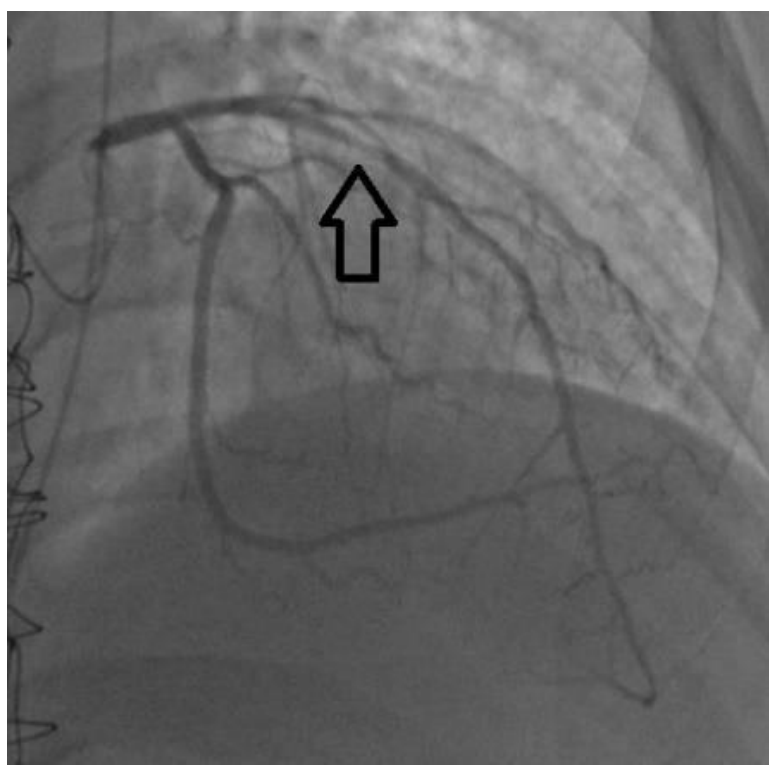

Figure 2: After administration of sublingual nitroglycerin, the stenoses marginally improved but slow flow throughout the coronary bed remained. Arrow shows slight improvement in mid left anterior descending stenosis.

molecular level, mediators including acetylcholine, serotonin, and histamine trigger the release of nitric oxide by endothelin resulting in vasodilation [1]. On the other hand, the same mediators stimulate vascular smooth muscle cells resulting in vasoconstriction. With endothelial dysfunction, the balancing mechanism is impaired and results in vasoconstriction and spasm [1].

Molecular pathways involved in CAS in acute OHT rejection are even more complex than CAS in non-transplanted patients. Factors contributing CAS in acute OHT rejection include complement deposition, impaired endothelial adhesion to extracellular matrix, lower nitric oxide production, and reduction of endothelium dependent relaxation to acetylcholine [3]. Furthermore, endothelial Notch ligand Dll4 is upregulated in AMR which induces macrophage polarization into a pro-inflammatory state and intravascular macrophage infiltrate. The overall result is microvascular inflammation [4].

Previously, Schlaifer and Mills compared the vasomotor response in epicardial coronary artery before and after immunosuppression with steroid in acute OHT rejection [15]. Impaired coronary blood flow response to acetylcholine was found at baseline in all nine subjects. More importantly, after three days of intense intravenous methylprednisolone therapy, repeat invasive study showed a trend towards greater dilation of epicardial coronary artery with administration of nitroglycerin. The study demonstrated that steroid therapy in acute OHT rejection reduced microvascular inflammation and improved epicardial coronary artery vasomotor response, which explains our observations in the presented case.

The current case report may also have important treatment implications in patients with CAS who are in acute OHT rejection. CAS can cause life threatening conditions including coronary artery occlusion, coronary ischemia, ventricular fibrillation, cardiac arrest $[11,14,16]$, and in our case acute decompensated systolic heart failure $[17,18]$. The CAS marginally improved with sublingual nitroglycerin but did not achieve complete distal flow normalization until patient received antirejection immunosuppressive therapy for acute OHT rejection with methylprednisolone. This suggests that patients in acute OHT rejection and severe CAS, treatment with vasodilators may not be adequate and may require the initiation of antirejection therapy for complete resolution.

\section{Conclusion}

We report the first case of CAS in acute $\mathrm{OHT}$ rejection with complete resolution of CAS and improved systolic heart failure by antirejection therapy with methylprednisolone. Furthermore, treatment response was immediate, within $24 \mathrm{~h}$. The proposed mechanism of action is reduced microvascular inflammation from steroid therapy resulting in enhanced vasomotor response, resolution of CAS, and improved

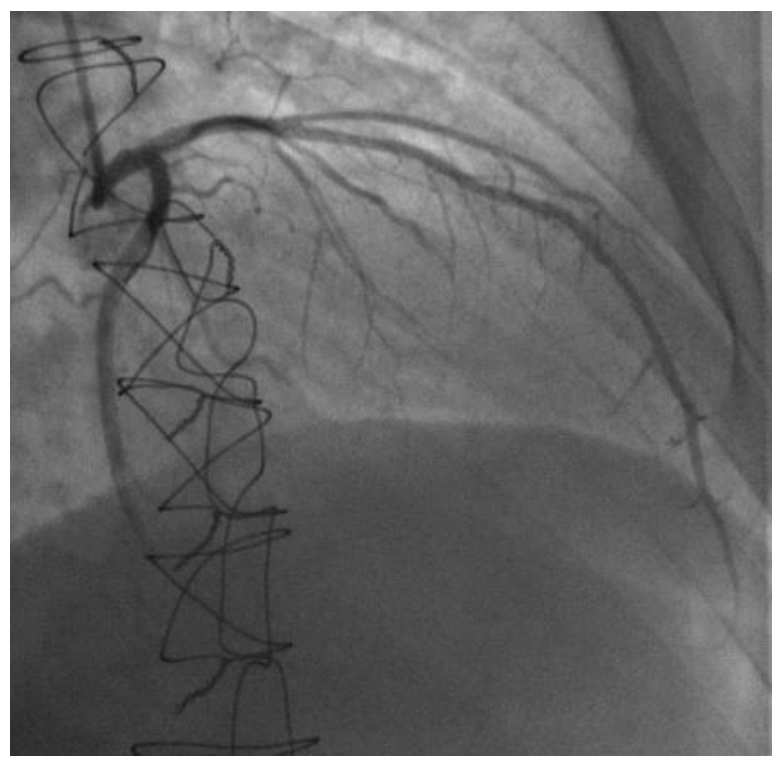

Figure 3: Right anterior oblique with cranial angulation view demonstration complete resolution of left anterior descending coronary artery spasm after one day of antirejection therapy with methylprednisolone. 
Citation: Lan H, Stoletniy L, Sakr A, Hilliard A (2016) Rapid Resolution of Coronary Artery Spasm Complicated by Acute Systolic Heart Failure with Antirejection Therapy in Acute Orthotopic Heart Transplant Rejection. Cardiovasc Pharm Open Access 5: 201. doi: 10.4172/23296607.1000201

Page 3 of 3

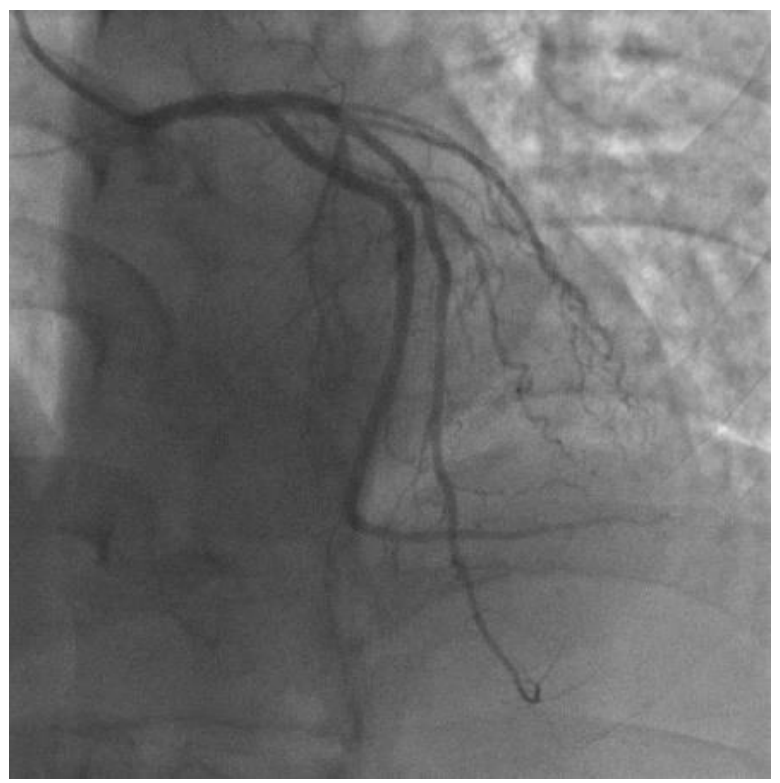

Figure 4: Left anterior oblique with cranial angulation view demonstration complete resolution of left anterior descending coronary artery spasm after one day of antirejection therapy with methylprednisolone.

hemodynamics. This case report suggests important treatment implications in patients with CAS and acute OHT rejection, especially if patient is in life threatening clinical situations including coronary artery occlusion, coronary ischemia, severe heart failure, or fatal arrhythmias. Early initiation of steroid therapy may help to improve CAS and could potentially serve as a lifesaving intervention.

\section{References}

1. Lanza GA, Careri G, Crea F (2011) Mechanisms of coronary artery spasm. Circulation 124: 1774-1782.

2. Bisognano JD, Lindenfeld J, Hammond E, Zisman LS (2005) Coronary artery vasospasm causing acute myocardial infarction in a heart transplant recipient. J Heart Lung Transplant 24: 355-358.

3. Cable DG, Hisamochi K, Schaff HV (1999) A model of xenograft hyperacute rejection attenuates endothelial nitric oxide production: a mechanism for graft vasospasm. J Heart Lung Transplant 18: 177-184.
4. Pabois A, Pagie S, Gérard N, Laboisse C, Pattier S, et al. (2016) Notch signaling mediates crosstalk between endothelial cells and macrophages via DII4 and IL6 in cardiac microvascular inflammation. Biochem Pharmacol 104 95-107.

5. Costanzo MR, Dipchand A, Starling R, Anderson A, Chan M, et al. (2010) The International Society of Heart and Lung Transplantation Guidelines for the care of heart transplant recipients. J Heart Lung Transplant 29: 914-956.

6. Auphan N, DiDonato JA, Rosette C, Helmberg A, Karin M (1995) Immunosuppression by glucocorticoids: inhibition of NF-kappa B activity through induction of I kappa B synthesis. Science 270: 286-290.

7. Snyder DS, Unanue ER (1982) Corticosteroids inhibit murine macrophage la expression and interleukin 1 production. J Immunol 129: 1803-1805.

8. Thrush PT, Pahl E, Naftel DC, Pruitt E, Everitt MD, et al. (2016) A multiinstitutional evaluation of antibody-mediated rejection utilizing the Pediatric Heart Transplant Study database: Incidence, therapies and outcomes. J Heart Lung Transplant 2498: 30192-30199.

9. Frist WH, Merrill WH, Eastburn TE, Atkinson JB, Stewart JR, et al. (1990) Unique antithymocyte serum versus OKT3 for induction immunotherapy after heart transplantation. J Heart Transplant 9: 489-494.

10. Uber WE, Uber LA, VanBakel AB, Crumbley AJ, Pereira NL, et al. (2004) CD3 monitoring and thymoglobulin therapy in cardiac transplantation: clinical outcomes and pharmacoeconomic implications. Transplant Proc 36: 3245-3249.

11. Buda AJ, Fowles RE, Schroeder JS, Hunt SA, Cipriano PR, et al. (1981) Coronary artery spasm in the denervated transplanted human heart: a clue to underlying mechanisms. Am J Med 70: 1144-1149.

12. Cattan S, Drobinski G, Artigou JY, Grogogeat Y, Cabrol C (1988) Coronary artery spasm in a transplant patient. Eur Heart J 9: 557-560.

13. Dalal JN, Brinker JA, Resar JR (1996) Coronary artery spasm in the transplanted human heart. A case report. Angiology 47: 291-294.

14. Goldenberg IF, Levine TB (1986) Coronary artery spasm in a denervated orthotopic transplanted human heart. Cathet Cardiovasc Diagn 12: 44-47.

15. Schlaifer JD, Mills RM (2000) Effect of low molecular weight heparin on coronary endothelial function in acute cellular heart transplant rejection. Am J Cardiol 86: 117-120

16. Romagnoli E, Lanza GA (2007) Acute myocardial infarction with norma coronary arteries: role of coronary artery spasm and arrhythmic complications. Int J Cardiol 117: 3-5.

17. Mancio J, Caeiro D, Faria R, Marques M, Bernardino S, et al. (2015) A 75-year-old woman with chest pain and transient severe left ventricular systolic dysfunction. Rev Port Cardiol 34: 621.

18. Oda S, Fujii Y, Takemoto H, Nomura S, Nakayama H, et al. (2014) Heart failure in which coronary spasms played an important role. Intern Med 53: 227-232. 